\title{
ANOTHER VISIT TO TWO HALFLINES
}

\author{
G. HOOGHIEMSTRA, ${ }^{*}$ Delft University of Technology
}

\begin{abstract}
We shall use three basic properties of Brownian motion to derive in an elegant and non-computational way the probability that standard Brownian motion, starting from 0 , will ever cross the halflines $t \rightarrow \alpha t+\beta$ or $t \rightarrow \gamma t+\delta$ where $\gamma, \delta<0<\alpha, \beta$.

BOUNDARY CROSSING; BROWNIAN MOTION; KOLMOGOROV-SMIRNOV STATISTICS
\end{abstract}

\section{Introduction}

In a famous paper Doob (1949) derived the distributions of the Kolmogorov-Smirnov statistics from the probability that standard Brownian motion $W(t)$, starting at the origin, will ever cross the halflines indicated above (see also Durbin (1973)). Here we present an elementary and non-computational derivation of this result based on the following three properties of Brownian motion that are included in every basic course on this subject:

(i) The stochastic process $\tilde{W}(t), t \geqq 0$, defined by

$$
\tilde{W}(t)= \begin{cases}0, & t=0 \\ t W\left(t^{-1}\right), & t>0\end{cases}
$$

is again standard Brownian motion.

(ii) The so-called scaling property: for each $\sigma>0$

$$
\sigma^{-1} W\left(\sigma^{2} \cdot\right)
$$

is again standard Brownian motion.

(iii) The explicit form of $g_{a, b}(u, y)$, the transition density for Brownian motion, started at 0 and killed on leaving $[b, a]$, where $b<0<a$ and $c=a-b$, given by

$$
\begin{aligned}
g_{a, b}(u, y) & =(2 \pi u)^{-\frac{1}{2}} \sum_{n=-\infty}^{\infty}\left\{\exp \left[-\frac{(y+2 n c)^{2}}{2 u}\right]-\exp \left[-\frac{(y-2 b+2 n c)^{2}}{2 u}\right]\right\} \\
& \left.=P\left\{b<\inf _{s<u} W(s) \leqq \sup _{s<u} W(s)<a, W(u) \in d y\right\} / d y\right\} / d y, y \in[b, a] .
\end{aligned}
$$

The precise result we shall prove is the following.

Theorem. For $\gamma, \delta<0<\alpha, \beta$

$$
\begin{gathered}
P\left\{\sup _{t \geqq 0}(W(t)-(\alpha t+\beta))<0, \inf _{t \geqq 0}(W(t)-(\gamma t+\delta))>0\right\} \\
=(2 \pi)^{\frac{1}{2}} \exp \left[\frac{1}{2}\left(\frac{\beta \gamma-\alpha \delta}{\sigma(\beta-\delta)}\right)^{2}\right] g_{\alpha / \sigma, \gamma / \sigma}\left(1, \frac{\beta \gamma-\alpha \delta}{\sigma(\beta-\delta)}\right),
\end{gathered}
$$

where $\sigma:=((\alpha-\gamma) /(\beta-\delta))^{\frac{1}{2}}$.

Received 15 May 1989; revision received 14 June 1989.

* Postal address: Faculty of Technical Mathematics and Informatics, Delft University of Technology, P.O. Box 356, 2600 AJ Delft, The Netherlands. 


\section{The derivation}

Part $u=1$ and replace $W$ by $\tilde{W}$ in (1.1) to obtain

$$
P\left\{b<\inf _{[0,1]} \tilde{W}(s) \leqq \sup _{[0,1]} \tilde{W}(s)<a, \tilde{W}(1) \in d y\right\}=g_{a, b}(1, y) d y .
$$

Since $\tilde{W}(0)=0$ a.s., we obtain

$$
P\left\{b<\inf _{(0,1]} s W\left(s^{-1}\right) \leqq \sup _{(0,1]} s W\left(s^{-1}\right)<a, W(1) \in d y\right\}=g_{a, b}(1, y) d y .
$$

We condition on the value of $W(1)$, which is a standard normal distributed random variable, and rewrite (2.2) as

$$
P\left\{b<\inf _{(0,1]} s W\left(s^{-1}\right) \leqq \sup _{(0,1]} s W\left(s^{-1}\right)<a \mid W(1)=y\right\}=(2 \pi)^{\frac{1}{2}} e^{y^{2} / 2} g_{a, b}(1, y) .
$$

For $s \in(0,1)$ we have $s^{-1} \geqq 1$ and so by the weak Markov property $\left\{W\left(s^{-1}\right)-W(1), s^{-1} \geqq 1\right\}$ is independent of $W(1)$. Hence the left-hand side of $(2.3)$ is equal to

$$
P\left\{b<\inf _{(0,1]}\left(s W\left(s^{-1}\right)-s W(1)+s y\right) \leqq \sup _{(0,1]}\left(s W\left(s^{-1}\right)-s W(1)+s y\right)<a\right\} .
$$

Furthermore $\left\{W\left(s^{-1}\right)-W(1), s^{-1} \geqq 1\right\}$ has the same distribution as $\left.\{W(1-s) / s), s^{-1} \geqq 1\right\}$, and so the simple time substitution $t=(1-s) / s$ shows that

$$
\begin{aligned}
& P\left\{\sup _{t \geq 0}(W(t)-(a t+a-y))<0, \inf _{t \geq 0}(W(t)-(b t+b-y))>0\right\} \\
& =(2 \pi)^{\frac{1}{2}} e^{y^{2} / 2} g_{a, b}(1, y) .
\end{aligned}
$$

Finally we take $\sigma=[(\alpha-\gamma) /(\beta-\delta)]^{\frac{1}{2}}, \quad a=\alpha / \sigma, \quad b=\gamma / \sigma, \quad y=(\gamma \beta-\alpha \delta) / \sigma(\beta-\delta) \epsilon$ $[\gamma / \sigma, \alpha / \sigma]$ and apply (ii) to obtain

$$
\begin{aligned}
& (2 \pi)^{\frac{1}{2}} e^{y^{2} / 2} g_{\alpha / \sigma, \gamma / \sigma}(1, y) \\
& =P\left(\sup _{t \geqq 0}\left(W(t)-\left(\frac{\alpha}{\sigma} t+\frac{\alpha}{\sigma}-y\right)\right)<0, \inf _{t \geqq 0}\left(W(t)-\left(\frac{\gamma}{\sigma} t+\frac{\gamma}{\sigma}-y\right)\right)>0\right\} \\
& =P\left\{\sup _{s \geqq 0}\left(\frac{1}{\sigma} W\left(\sigma^{2} s\right)-\left(\alpha s+\frac{\alpha}{\sigma^{2}}-\frac{y}{\sigma}\right)\right)<0, \inf _{s \geqq 0}\left(\frac{1}{\sigma} W\left(\sigma^{2} s\right)-\left(\gamma s+\frac{y}{\sigma^{2}}-\frac{y}{\sigma}\right)\right)>0\right\} \\
& =P\left\{\sup _{s \geqq 0}\left(W(s)-\left(\alpha s+\frac{\alpha}{\sigma^{2}}-\frac{y}{\sigma}\right)\right)<0, \inf _{s \geqq 0}\left(W(s)-\left(\gamma s+\frac{\gamma}{\sigma^{2}}-\frac{y}{\sigma}\right)\right)>0\right\},
\end{aligned}
$$

which yields (1.2) after verifying that $y=\alpha / \sigma-\beta \sigma=\gamma / \sigma-\delta \sigma$.

Relation (2.5) was also noted in Hooghiemstra (1987), but there the proof was a bit more involved. Note that (2.3) together with (2.4) is sufficient for the most general KolmogorovSmirnov statistic, because it implies (once more using (i)),

$$
\begin{aligned}
P & \left\{b<\inf _{[0,1]}(W(t)-t W(1)) \leqq \sup _{[0,1]}(W(t)-t W(1))<a\right\} \\
& =P\left\{b<\inf _{[0,1]}(\tilde{W}(t)-t \tilde{W}(1)) \leqq \sup _{[0,1]}(\tilde{W}(t)-t \tilde{W}(1))<a\right\} \\
& =P\left\{b<\inf _{(0,1]} t\left(W\left(\frac{1}{t}\right)-W(1)\right) \leqq \sup _{(0,1]} t\left(W\left(\frac{1}{t}\right)-W(1)\right)<a\right\} \\
& =(2 \pi)^{\frac{1}{2}} g_{a, b}(1,0) .
\end{aligned}
$$




\section{References}

Doob, J. L. (1949) Heuristic approach to the Kolmogorov-Smirnov theorems. Ann. Math. Statist. 20, 393-403.

Durbin, J. (1973) Distribution Theory for Test Based on the Sample Distribution Function. SIAM, Philadelphia.

Hooghiemstra, G. (1987) On functionals of the adjusted range process. J. Appl. Prob. 24, 252-257. 